\title{
Neutralidad en la red en Estados Unidos: De vuelta al pizarrón
}

Net neutrality in the United States: Back to the drawing board

MARTÍN MOIS FREIWIRTH

Abogado, Aninat Schwencke \& Cía

RESUMEN Este artículo analiza la sentencia de la Corte de Apelaciones del Circuito del Distrito de Columbia (el «Circuito D.C.») que anuló parcialmente la Open Internet Order, dictada por la Federal Communications Commission (FCC) de los Estados Unidos el 20ro, que había consagrado normativamente el principio de neutralidad de Internet. Para ello, junto con un breve repaso actual del tema, se revisan los antecedentes judiciales y administrativos previos a la sentencia relacionados con el principio de neutralidad. Luego se analizan los argumentos del Circuito D.C. para anular casi totalmente la Open Internet Order, incluyendo un breve comentario sobre el voto disidente. El artículo termina con conclusiones relativas a la sentencia y a las eventuales decisiones que ahora podría tomar la FCC.

PALABRAS CLAVE Circuito D.C., common carrier, FCC, neutralidad de Internet, Orden de Internet Abierta, Verizon.

ABSTRACT This paper analyzes the United States Court of Appeals for the District of Columbia Circuit's («D.C. Circuit») opinion that partially vacated the Federal Communications Commission's Open Internet Order of 20I0, which set the Network Neutrality Principle as a general norm. 
Starting with a brief overview of the issue, the judicial and administrative precedents related to the Network Neutrality Principle are reviewed. Then the D.C. Circuit's reasoning for vacating a large part of the Open Internet Order are analyzed, including a brief commentary on the dissent. The paper ends with conclusions regarding the opinion and eventual entailing decisions by the FCC.

KEYWORDS D.C. Circuit, common carrier, FCC, Network Neutrality, Open Internet Order, Verizon.

\section{INTRODUCCIÓN}

Al parecer, 20I4 será el año de la neutralidad de Internet. En lo que va de este año varias noticias han visibilizado nuevamente la verdadera batalla que se libra en Estados Unidos a favor y en contra del reconocimiento normativo del principio de neutralidad: la anunciada absorción de la empresa Time Warner Cable por la empresa Comcast ${ }^{1}$ (lo que consolidará aún más en Estados Unidos el mercado de acceso a Internet), el acuerdo entre Netflix y la misma empresa Comcast, y también con la empresa Verizon, por el cual Netflix le pagará a esos proveedores de acceso a Internet una tarifa especial para darle acceso directo a sus suscriptores $^{2}$ y, por supuesto, la sentencia de la Corte de Apelaciones del Circuito del Distrito de Columbia (el «Circuito D.C.») ${ }^{3}$ del I 4 de enero

I. «Comcast anuncia la compra de Time Warner Cable por US\$45.200 millones», en Pulso, 27 de febrero de 20I4, disponible en <http://www.pulso.cl/noticia/empresa-mercado/internacional/20 I 4/02/72-384 I 4-9-comcast-anuncia-la-compra-de-time-warnercable-por-us45200-millones.shtml>.

2. E. Wyatt y N. Cohen, «Comcast and Netflix Reach Deal on Service», en The New York Times, 23 de febrero de 20I 4, disponible en <http://www.nytimes.com/20I 4/02/24/ business/media/comcast-and-netflix-reach-a-streaming-agreement.html>. Este acuerdo, conocido como "paid peering», le permitirá a Netflix conectarse directamente con los servidores de Comcast sin pasar por intermediarios. A fines de abril, Netflix anunció un acuerdo similar con Verizon: «Netflix pays Verizon in streaming deal, following Comcast pact», disponible en <http://time.com/80I92/netflix-verizon-paid-peering-agreement/>.

3. Como «Circuito D.C.» («D.C. Circuit») se conoce a la Corte de Apelaciones del Circuito del Distrito de Columbia, con sede en la ciudad de Washington D.C. Es la corte federal de apelaciones para los tribunales federales del Distrito de Columbia. Como to- 
de este año, que invalidó parcialmente la Open Internet Order de 20I0, dictada por la Federal Communications Commission (FCC) de Estados Unidos, el año 2010. ${ }^{4}$

La sentencia del Circuito D.C. viene a ser el más reciente capítulo de una telenovela regulatoria que tiene como gran protagonista a la FCC, y como antagonista a los proveedores de acceso a Internet, en un tira y afloja que hasta ahora ha impedido que en Estados Unidos exista un reconocimiento normativo general al principio de neutralidad de Internet, considerando que han pasado ya más de diez años desde que el concepto fue acuñado (Wu, 2003). Y si bien en este juicio hay muchos temas relevantes respecto de los cuales el Circuito D.C. se ha pronunciado, aún está por verse si varios de los argumentos de fondo de empresas como Verizon, que impugnaron en este caso la Open Internet Order y que permanentemente buscan la eliminación de cualquier norma que huela a imposición del principio, sirven para fundamentar el que el principio no se reconozca normativamente.

Lo relevante de esta sentencia, más allá de sus consecuencias, es el rayado de cancha administrativo que el Circuito D.C. le hizo a la FCC. Por ello, quienes busquen en esta sentencia una fundamentación detallada sobre la legalidad de imponer normativamente el principio de neutralidad, o los argumentos para justificar el que dicho principio no tenga reconocimiento legal, tendrán que seguir esperando: esta sentencia toca principalmente la pregunta de si la FCC estaba legalmente facultada para dictar algunas de las normas de la Open Internet Order a partir de las atribuciones que la Ley de Telecomunicaciones de los Estados Unidos ${ }^{5}$ le otorga. Ello, sin embargo, no deja de ser interesante para el estudio de este tema, pues la justificación fáctica-económica que usó la FCC para dictar inicialmente la Open Internet Order es probablemente uno de los

das las cortes de apelaciones federales de Estados Unidos, sus sentencias sólo pueden ser revisadas por la Corte Suprema.

4. Ver Verizon v. FCC, No. II-I355, slip op. at I9-27 (D.C. Cir. Jan. I4, 20I4), sentencia completa disponible en <http://www.cadc.uscourts.gov/internet/opinions.nsf/3 $\mathrm{AF}$ 8B4D938CDEEA68 5257C6000532062/\$file/I I-I355-I 474943.pdf>. Sentencia dictada por tribunal conformado por los jueces David S. Tatel, Judith W. Rogers y Laurence H. Silberman.

5. Véase el texto completo de la ley, Título 47 del U.S. Code, disponible en $<$ http:// www.law.cornell.edu/uscode/text/47>. 
temas más discutidos entre los bandos que luchan por la neutralidad de Internet o, contrariamente, por la diversidad de redes.

Por ello, vale la pena efectuar un breve resumen histórico de este principio en Estados Unidos, antes de referirse a la sentencia propiamente tal.

\section{ANTECEDENTES}

El propio Circuito D.C. en la sentencia que comentamos se preocupa de hacer un resumen exhaustivo de este verdadero gallito regulatorio que ha significado el principio de neutralidad de Internet y su reconocimiento normativo en Estados Unidos.

La primera vez que la FCC empleó sus atribuciones para eventualmente llegar a regular Internet, y ello fue autorizado por los tribunales, se encuentra en los actos administrativos de la FCC conocidos como Computer Inquiries (Cannon, 2003), y especialmente la segunda investigación de I980, conocida como Computer II, en que la FCC distinguió los servicios «básicos» de los servicios «mejorados» provistos por las empresas de telecomunicaciones reguladas por la Ley de Comunicaciones de Estados Unidos de 1934: el criterio para distinguir era si los servicios prestados, además de ser netamente de transmisión (básicos), también realizaban procesamiento de datos (mejorados). A partir de dicho criterio, la FCC obligó a las empresas de telecomunicaciones a no discriminar en la provisión de servicios básicos, sin regular a aquellas que prestaran servicios mejorados.

El año I996, el Congreso de Estados Unidos dictó una nueva Ley de Telecomunicaciones (la «Ley I996»), modificando la Ley de Comunicaciones de I934, en la que se distinguen dos categorías de entidades reguladas: los carriers de telecomunicaciones (aquellas empresas que proveen el servicio básico, es decir, sólo transmiten), y los proveedores de servicios de información (aquellas empresas que proveen el servicio mejorado, es decir, efectúan procesamiento de datos). La Ley 1996 estableció para los carriers de telecomunicaciones, pero no para los proveedores de servicios de información, las obligaciones de common carrier (según se define más adelante) establecidas en el título II de la ley.

Esta distinción ha sido la causa de los conflictos entre la FCC y sus entes regulados, a partir de decisiones posteriores que la FCC tomó y que tendrían una dudosa justificación fáctica y jurídica. El 2002, la FCC dic- 
tó una norma declarativa y propuesta de norma relativa al tratamiento que se le daría al acceso a Internet a través del cable (la Cable Broadband Order) (FCC, 2002), en la que declaró que los proveedores de servicio de banda ancha (y todos los servicios que prestan, incluyendo transmisión pura de datos) no eran carriers de telecomunicaciones, sino proveedores de servicios de información, con lo cual estas empresas quedaron exentas de las obligaciones de common carrier a que están sujetas las empresas de telecomunicaciones que efectúan solamente transmisión, y no procesamiento de datos. La legalidad de esta decisión de la FCC fue confirmada por la Corte Suprema de Estados Unidos el año 2005, en la sentencia conocida como Brand X, ${ }^{6}$ por la cual la Corte Suprema resolvió que el sistema de clasificación de la FCC a partir de su interpretación de lo que era un servicio de telecomunicaciones era razonable, aunque entraba en conflicto con su interpretación anterior. Brand $X$ sirvió para que la FCC clasificara también a los servicios de acceso a Internet a través de DSL y vía teléfonos celulares como proveedores de servicios de información, clasificación que se mantiene hasta el día de hoy a pesar de ser severamente criticada, pues es de hecho uno de los fundamentos por los cuales aún no pueden consagrarse normativamente los principios de neutralidad de Internet.

El año 2005 apareció la primera mención regulatoria propiamente tal a lo que básicamente es la neutralidad de la red, contenida en una Declaración de Política Pública, ${ }^{7}$ en que la FCC amenazó con regular a los

6. Véase National Cable \& Telecommunications Ass'n v. Brand X Internet Services, 545 U.S. 967 (2005), disponible en <http://www.law.cornell.edu/supct/html/o4-277. ZS.html>.

7. Ver FCC, Policy Statement 07-I 5 I, del 5 de agosto de 2005, disponible en <http:// hraunfoss.fcc.gov/edocs_public/attachmatch/FCC-05-I 5 IAI.pdf>. Los cuatro principios adoptados en esta Declaración son (traducción del autor, itálicas en el original):

Para incentivar el despliegue de banda ancha y preservar y promover la naturaleza abierta e interconectada de la Internet pública, los consumidores tienen derecho a acceder al contenido legal de Internet que elijan.

Para incentivar el despliegue de banda ancha y preservar y promover la naturaleza abierta e interconectada de la Internet pública, los consumidores tienen derecho a ejecutar las aplicaciones y usar los servicios de su elección, sujetos a las necesidades de cumplimiento de las leyes.

Para incentivar el despliegue de banda ancha y preservar y promover la naturaleza 
proveedores de acceso a Internet (considerados como servicios de información) en caso de existir evidencia de que éstos violasen los principios de la naturaleza abierta e interconectada de Internet. El 2008, a raíz de una denuncia de clientes de la empresa de televisión por cable y servicios de Internet Comcast, afirmando que ésta infringía dicha Declaración, y acusándola de degradar la calidad del tráfico de aplicaciones peer-topeer, la FCC dictó una orden obligando a Comcast a establecer una nueva política respecto de la forma de administrar la demanda por su ancho de banda, así como a hacer pública dicha nueva política. La FCC justificó sus atribuciones para dictar esta orden en lo que se conoce como su «jurisdicción supletoria» (ancillary jurisdiction), esto es, la atribución de la FCC para realizar todos los actos, dictar todas las reglas y normas, y emitir todas las órdenes, que no contradigan la misma norma que le otorga esa atribución, y que sean necesarias para ejecutar sus funciones. ${ }^{8}$ Sin embargo, Comcast demandó ante el Circuito D.C. la anulación de la orden en su contra, alegando que la FCC no tenía atribuciones para dictarla. En la sentencia del caso Comcast de 20ro, el Circuito D.C. le dio la razón a dicha empresa, declarando que la FCC no había demostrado que su jurisdicción supletoria era suficiente para obligar a Comcast a no efectuar gestión de tráfico que degradara las aplicaciones peer-to-peer. ${ }^{9}$ Uno de los argumentos que dio el tribunal, que es sumamente relevante para lo que vendría, es que la forma que tenía la FCC para someter a empresas como Comcast a su jurisdicción principal (y sujetarlos al régi-

abierta e interconectada de la Internet pública, los consumidores tienen derecho a conectar libremente aparatos lícitos que no dañen la red.

Para incentivar el despliegue de banda ancha y preservar y promover la naturaleza abierta e interconectada de la Internet pública, los consumidores tienen derecho a que exista competencia entre los proveedores de redes, de aplicaciones y de servicios, y los proveedores de contenidos.

8. Letra (i) de la sección I 54 del capítulo 5 del título 47 del U.S. Code, disponible en <http://www.law.cornell.edu/uscode/text/47/I 54>, proviene de la Ley de Comunicaciones de 1934 .

9. Comcast Corp. v. FCC, 600 F. 3d 642 (D.C. Cir. 2010), sentencia disponible en $<$ http://scholar.google.com/scholar_case?case=I 2 I 5870566 I002658248>. En la sentencia, el Circuito D.C. resolvió que la FCC no pudo demostrar que el acto efectuado (prohibirle a Comcast el poder interferir con el uso que sus suscriptores hacen de aplicaciones peer-to-peer) podía autorizarse en virtud de su autoridad supletoria. 
men de common carrier) era reclasificar los servicios prestados por estas empresas de forma tal que no fueran considerados servicios de información, sino servicios de transmisión.

Pero para la fecha en que el Circuito D.C. resolvió el caso Comcast, la FCC ya se encontraba trabajando en una nueva norma de política pública, que finalmente fue dictada el año 20 Io con el nombre de Open Internet Order ( Orden de Internet Abierta» o OIA). ${ }^{10}$ El 20 I I el ISP Verizon solicitó al Circuito D.C. la revisión judicial de esta nueva orden.

\section{ANÁLISIS}

Como indicamos anteriormente, la sentencia del Circuito D.C. se refiere principalmente a si la justificación que invocó la FCC para dictar la OIA se encuentra respaldada por las propias normas que regulan sus funciones. Por lo tanto, y aunque fueron argumentadas por Verizon, defensas de fondo y que son doctrinariamente reconocidas como fundamentos relevantes para rechazar el reconocimiento normativo del principio de neutralidad, no fueron analizadas por el Circuito D.C. al estimar que la litis se resolvía a partir de si la FCC tenía o no atribuciones para regular a los proveedores de servicios de Internet (ISP) como lo hizo con la OIA. Por ejemplo, Verizon en su demanda planteó que la OIA infringía su derecho de libre expresión bajo la Primera Enmienda, argumento que se fundamenta en la rica y nutrida jurisprudencia de la Corte Suprema de Estados Unidos respecto a la discreción editorial que ampara a las empresas de comunicaciones (cf. Yoo, 20I3a). Igualmente, Verizon señaló que la imposición de la OIA constituía una expropiación por causa de utilidad pública encubierta que no cumplía con la Quinta Enmienda, argumento relevante a partir del derecho de propiedad ${ }^{11}$ y respecto del

Io. Una copia completa de la OIA se encuentra disponible en $<$ http://hraunfoss.fcc. gov/edocs_public/attachmatch/FCC-IO-20IAI_Rcd.pdf>.

II. En especial la llamada Cláusula de Incautación de la Quinta Enmienda que, al igual que el artículo I9 núm. 24 inciso tercero de la Constitución, garantiza que nadie podrá ser privado de su propiedad, sino en virtud de ley general o especial que autorice la expropiación por causa de utilidad pública, e indemnizando al propietario por el daño patrimonial efectivamente causado. Sobre la doctrina referente a que el reconocimiento normativo del principio de neutralidad resultaría en una afección al derecho de propiedad del ISP, véase por ejemplo Owen y Rosston (2006). 
cual, por lo menos en relación con la neutralidad de Internet, no ha sido objeto de un análisis judicial profundo.

El Circuito D.C. inicia su análisis de la OIA describiendo las normas que en ella se contemplan para dos regímenes separados, distinguiendo entre prestadores de servicio de banda ancha fija y prestadores de servicio de banda ancha móvil. Luego, resalta que las normas de la OIA son de tres clases: normas de transparencia y de antibloqueo (aplicables a ambos regímenes) y de antidiscriminación (aplicables solamente al régimen de prestadores de acceso a Internet fija). La similitud de los tres tipos de normas con las disposiciones de la Ley $20.453^{12}$ son evidentes, todas utilizan básicamente el mismo lenguaje de nuestra ley:

- Respecto de las normas de transparencia, se obliga a los ISP, tanto aquellos que ofrezcan acceso a Internet vía banda ancha fija como vía banda ancha móvil, a revelar públicamente información precisa respecto de la administración de sus redes en lo que dice relación con sus prácticas, rendimiento y términos comerciales de acceso, que sean suficientes para que los consumidores tomen decisiones informadas respecto del uso de dichos servicios y para que los proveedores de contenidos, aplicaciones, servicios y aparatos desarrollen, comercialicen y mantengan ofertas en Internet. $^{13}$

- Respecto de las normas antibloqueo, se obliga a quienes provean acceso a Internet: I) Vía banda ancha fija, a no bloquear contenidos, aplicaciones y servicios legales, ni el uso de periféricos inocuos, sin perjuicio de una administración razonable de sus redes. ${ }^{14}$ 2) Vía banda ancha móvil, a no bloquear el acceso de los usuarios a sitios web, sujeto a administración razonable de la red; ni a bloquear aplicaciones que compitan con los servicios de voz

I2. La Ley 20.453, publicada en el Diario Oficial el 26 de agosto de 2010, consagró el principio de neutralidad en la red para los consumidores y usuarios de Internet. Fue la primera norma de rango legal de su tipo promulgada en el mundo, y establece para los proveedores de acceso a Internet obligaciones muy similares a las de la OIA, aunque sin distinguir entre proveedores de acceso fijo y proveedores de acceso móvil.

I3. OIA, cit. nota I3, p. I7936, para proveedores de acceso fijo, y p. I7.958, para proveedores de acceso móvil.

I4. OIA, cit. nota I3, pp. I7.94I-I7.942. 
o videotelefonía del proveedor, sujeto a administración razonable de la red. ${ }^{15}$

- Respecto de las normas antidiscriminación, se prohíbe a quienes provean acceso a Internet vía banda ancha fija, discriminar de forma no razonable en la transmisión de tráfico legal en la red en el servicio de acceso a Internet de banda ancha del consumidor. Pero una administración razonable de la red no constituirá una discriminación no razonable. ${ }^{16}$

Para verificar si la interpretación que la FCC efectuó de sus propias atribuciones para dictar la OIA era o no legítima, el Circuito D.C. aplicó el test Chevron. Desarrollado por la Corte Suprema de Estados Unidos en $1984,{ }^{17}$ dicho test busca revisar la legalidad de la interpretación que un ente administrativo efectúa de las normas que lo regulan, y si su interpretación es legítima se respeta dicha interpretación. Este estándar de revisión judicial es una de las bases del derecho administrativo estadounidense y, como normalmente ocurre con estos estándares judiciales, existen dos requisitos que deben cumplirse para concluir si el acto administrativo conculcado es legal o no. En este caso, éstos son:

- Si el Congreso al dictar la ley se pronunció específicamente sobre el asunto cuestionado. Si la intención del Congreso es clara, el ente administrativo deberá actuar en base a esa intención. Pero si el Congreso no se pronunció específicamente sobre el asunto cuestionado, entonces se aplica el segundo paso:

- Si la norma es ambigua u omite el asunto, entonces se analiza si la aplicación de la norma por el ente administrativo está basada en una interpretación razonable de la misma. Si lo está, el tribunal preferirá esa interpretación aunque pueda no estar de acuerdo con ella.

El Circuito D.C. aplicó el test Chevron, revisando respecto de cada argumento de las partes cómo calzaban con los requisitos del test.

\footnotetext{
I 5. OIA, cit. nota I3, p. I7.959.

I6. OIA, cit. nota I3, p. I7.944.

17. Chevron, U.S.A., Inc. v. Natural Resources Defense Council, Inc., 467 U.S. 837 (I984). Copia de la sentencia disponible en <https://bulk.resource.org/courts.gov/c/ US/467/467.US.837.82-I 591.82-I 247.82-1005.html>.
} 
En primera instancia, y en términos generales, la FCC justificó la dictación de la OIA en las atribuciones que se le confieren en las secciones 706 (a) y 706 (b) del título VII de la Ley i996. Específicamente, la sección 706 trata sobre los incentivos para el desarrollo de servicios de telecomunicaciones avanzadas. En su letra a) dispone en general que la FCC tiene jurisdicción para promover el despliegue de capacidad de telecomunicación avanzada para todos los estadounidenses, de forma razonable y oportuna, utilizando, de forma compatible con el interés, conveniencia y necesidad del público, límites de precios, exención de normas, medidas que promuevan la competencia en el mercado local de telecomunicaciones, y otros métodos regulatorios que eliminen barreras para inversión en infraestructura. Luego, en su letra b) dispone que la FCC podrá periódicamente iniciar procesos de investigación respecto de la capacidad de telecomunicaciones avanzadas para todos los estadounidenses. En dichos procesos determinará si la capacidad de telecomunicaciones avanzadas está siendo desplegada para todos los estadounidenses de forma razonable y oportuna. Si la conclusión de la FCC es negativa, tomará inmediatamente medidas para acelerar el despliegue de dicha capacidad eliminando barreras para la inversión en infraestructura y promoviendo la competencia en el mercado de las telecomunicaciones.

Respecto de la sección 706 (a), la FCC argumentó que ella le otorgaba facultades para dictar la OIA. Aunque el Circuito D.C. rechazó inicialmente este argumento en atención al precedente establecido en la revisión que hizo de la Advanced Services Order dictada por la FCC en I998, también reconoció que el propio test Chevron le permite al ente administrativo cambiar su interpretación de la norma siempre y cuando ello tenga fundamento. La FCC cambió su interpretación de la sección 706 (a) señalando que ésta le otorga autoridad normativa. El Circuito D.C. concluyó que la historia legislativa de la norma permitía interpretar que, efectivamente, la intención del Congreso fue darle autoridad normativa a la FCC, aunque dicha autoridad no es ilimitada: está restringida por una lectura armónica de otras normas de la ley, incluyendo: a) la necesidad de que el ámbito jurisdiccional esté circunscrito a comunicaciones por cable y radio interestatal y en el extranjero, y b) la necesidad de que toda norma dictada cumpla con un fin específico: el promover el despliegue de forma razonable y oportuna, de capacidad de telecomunicaciones avanzadas para todos los estadounidenses. 
Respecto de la sección 706 (b), es interesante destacar que, hasta el año 20Io, la FCC nunca había regulado en base a esta sección, por haber estimado en sus procesos investigativos anteriores, siempre, que el despliegue de infraestructura de telecomunicaciones avanzadas para todos los estadounidenses había sido razonable y oportuno. Pero el año 20I0, en su Sexto Informe sobre Despliegue de Banda Ancha, ${ }^{18}$ la FCC analizó el estado actual de Internet y concluyó que el cambio de hábitos de sus usuarios, que para ese entonces ya requerían de un ancho de banda suficiente para descargar video de forma predominante, hacía necesario reclasificar el límite mínimo de lo que se consideraba banda ancha. Así, el piso mínimo pasó de $200 \mathrm{Kbps}^{19}$ a 4 Mbps de descarga y I Mbps de subida el 20 Io.

Al aplicar dicho criterio, la FCC concluyó que más de 80 millones de estadounidenses no son suscriptores de banda ancha, y entre I4 a 24 millones ni siquiera tienen acceso a ella. La FCC invocó este hecho para utilizar las atribuciones conferidas por la sección 706 (b) para dictar la OIA con miras a acelerar el despliegue de infraestructura de banda ancha, estimando que lo hacía de forma oportuna y razonable. El Circuito D.C. acogió este argumento de la FCC, concluyendo que su interpretación de la norma era razonable.

Habiendo aceptado las atribuciones que la FCC argumentó tener para dictar las imposiciones a los ISP en la OIA, el Circuito D.C. revisó luego su racionalidad, a partir de los fundamentos contenidos en la misma y a la contestación de la FCC a la demanda de Verizon, que alegó que la FCC no tiene facultades para dictar la OIA, que su decisión de imponerla era arbitraria y caprichosa, y que la OIA infringe una prohibición expresa de la Ley I996 impidiéndole tratar a los ISP como common carriers. ${ }^{20}$

Respecto de la imposición de la OIA, su caracterización como «ar-

I8. Sección de la página web de la FCC sobre el Sexto Informe sobre Despliegue de Banda Ancha: <http://www.fcc.gov/reports/sixth-broadband-progress-report>. Vínculo al informe: <http://hraunfoss.fcc.gov/edocs_public/attachmatch/FCC-Io-I29AI_Rcd.pdf >.

19. En su Primer Informe sobre Despliegue de Banda Ancha, de 1999. Antecedentes disponibles en <https://www.fcc.gov/reports/first-broadband-progress-report>.

20. Como ya se mencionó, Verizon también alegó afectación de su libertad de expresión y de su derecho de propiedad, pero dichos argumentos no fueron analizados por la Corte por considerarlo innecesario. 
bitraria y caprichosa» por Verizon nace tanto del precedente Chevron como de las normas de la Administrative Procedure Act, ${ }^{21}$ ley que regula el procedimiento de propuesta y dictación de normas que deben seguir las agencias federales. Verizon argumentó que esta arbitrariedad proviene de los fundamentos lógicos y fácticos empleados por la FCC para dictar la OIA, en lo que —en términos del billar- denominó como un «tiro a tres bandas» (y que la FCC denominó como un «círculo virtuoso»). La imposición regulatoria se justificaría en que ella:

- protegerá y promoverá la inversión y desarrollo de proveedores de contenidos, servicios y aplicaciones en Internet (en la sentencia son calificados como edge providers, dando la FCC ejemplos como Amazon o Google);22

- ello a su vez aumentará la demanda de los usuarios finales de más y mejores tecnologías de banda ancha;

- lo que a su vez estimulará la competencia entre los proveedores de banda ancha para aumentar sus inversiones en su infraestructura.

Sobre estos argumentos, el Circuito D.C. le dio la razón a la FCC, afirmando que las siguientes conclusiones de la FCC estaban debidamente fundamentadas y explicadas:

- su pronóstico respecto a que la innovación por los edge providers resultaría en una ampliación y mejora de la infraestructura de banda ancha;

- su conclusión de que una Internet abierta promueve la innovación por los edge providers, lo que alimenta el círculo virtuoso;

- su conclusión de que a menos que se impongan normas como la OIA, los ISP amenazarían el carácter abierto de Internet al actuar de forma tal de inhibir la velocidad y alcance del despliegue futuro de banda ancha; $y$

- su conclusión de que, frente a los beneficios de la OIA, los costos asociados con la mayor regulación son bajos.

\footnotetext{
2I. Ver en $\langle$ http://www.law.cornell.edu/uscode/text/5/part-I/chapter-5>.

22. Verizon $v$. FCC, citado en nota 4, p. 5 .
} 
Common carriers. Pero a continuación, el Circuito D.C. analizó el argumento de Verizon alegando que la FCC por medio de la OIA le impone obligaciones de common carrier a los ISP sin estar legalmente facultada para ello. Es esta parte de la sentencia la más relevante, y vale la pena detenerse en ella porque las conclusiones de la Corte son la guía a través de la cual la FCC eventualmente podrá reconocer normativamente el principio de neutralidad evitando en lo posible nuevos desafíos judiciales a sus normas. En resumidas cuentas, la normativa de common carrier, ${ }^{23}$ que surgió originalmente vía precedente para luego reconocerse primeramente en la Ley Mann Elkins de I9ıo, y luego en el texto de la ley de $1934,{ }^{24}$ contempla obligaciones especiales para aquellos carriers que queden sujetos a dicho régimen, las que son más gravosas que las obligaciones generales de la ley. Por lo mismo, el fundamento legal empleado por la agencia regulatoria para justificar la imposición de este régimen es analizado detalladamente.

¿Qué es un common carrier? El Circuito D.C. comenta que no existe una buena definición en la ley, señalando que puede formarse una definición a partir del desarrollo jurisprudencial y normativo del concepto, y que la cualidad esencial de un common carrier es su obligación de servir al público sin poder discriminar. En el ámbito de las telecomunicaciones, la acepción de common carrier relevante fue dada en los casos Midwest Video $I I^{25}$ y Cellco, ${ }^{26}$ y a partir de dichos casos el Circuito D.C. obtiene los principios que guían su análisis en este caso, a saber: ${ }^{27}$

- Si un carrier es obligado a ofrecer sus servicios de forma indiscriminada y en términos generales, debe considerarse como common carrier.

23. El concepto de common carrier surgió inicialmente en el Reino Unido en el siglo $\mathrm{XV}$, de la necesidad de regular a empresas privadas que estaban realizando funciones que normalmente son públicas, como la administración de caminos, vías férreas, etc.

24. El título II de la Ley 1934 (que aún está vigente) define malamente common carrier como «toda persona que como common carrier preste servicios para comunicación interestatal o foránea por cable o radio, o de transmisión de energía interestatal o foránea por radio» (la traducción es nuestra).

25. FCC v. Midwest Video Corp., 440 U.S. 689 (I979).

26. Cellco Partnership v. FCC, 700 F. 3 d 534 (D.C. Cir. 20I2).

27. Verizon v. FCC, cit. nota 4, pp. 49-50. 
- Pero esta caracterización no es absoluta, pues cabe la posibilidad de que ciertas obligaciones impuestas al carrier sean propias de este régimen especial, sin que el regulado en sí se le confiera el estatus de common carrier (es decir, sin que sean obligaciones per se de common carrier)..$^{28}$

Es respecto de estos argumentos donde la defensa de la FCC falla, pues intenta un malabarismo legal argumentando que la OIA no impone obligaciones de common carrier para los ISP a pesar de que en la práctica lo hace. Esta necesidad surge porque la limitación a dicha regulación era algo que la propia FCC había propiciado: recordemos que en la Cable Broadband Order del 2000, la FCC había clasificado a los ISP como proveedores de servicios de información, y no carriers de telecomunicaciones, y que dicha clasificación fue confirmada por la Corte Suprema en el caso Brand X. Al efectuar esa clasificación, la FCC dejó fuera del régimen de common carrier a los proveedores de banda ancha, los que sólo podían ser regulados de esa forma en tanto ofrecieran servicios de transmisión (como el teléfono) y no de información. Así:

- La FCC argumenta que la OIA no impone normas de common carrier porque no obliga a los ISP respecto de sus usuarios finales, sino respecto de los edge providers: si obligara a servir a los usuarios finales de forma indiscriminada, entonces se aplicaría el régimen especial. El Circuito D.C. rechaza este argumento, concluyendo que respecto de los edge providers los ISP sí deben ser considerados como carriers, porque lo relevante son las obligaciones impuestas al proveedor independientemente de quienes sean sus principales clientes. $\mathrm{O}$ dicho de otra forma: la pregunta es si,

28. Según Christopher Yoo, existen cuatro situaciones de hecho que identifican lo que puede considerarse un common carrier, a partir de diversas sentencias que se han pronunciado sobre esta institución: I) disponibilidad: de servir a todo usuario que lo requiera sin discriminar; 2) sujeción a un interés público; 3) tener poder monopólico, o 4) transmisión sin transformación: esto es, que el proveedor de servicios de telecomunicaciones transmite los contenidos de sus usuarios de forma «pura» y sin intervenirlos (vía generación, adquisición, almacenamiento, transformación, procesamiento, recuperación, uso o puesta a disposición de la información, todos actos que definen lo que la Ley entiende como servicios de información, ver numeral I). Cf. Yoo, 20I3b. 
dadas las reglas de la OIA, los ISP están ahora obligados a actuar como common carriers. ${ }^{29}$

- Frente a esa pregunta, la respuesta de la FCC es negativa, y se ampara en varios argumentos, todos los que son desechados por la Corte..$^{30}$ La FCC intenta también argumentar que el precedente de Midwest Video $I^{31}$ no es aplicable, lo que también es desechado por la Corte. ${ }^{32}$ El Circuito D.C., luego de descartar estas defensas de la FCC, llega a la pregunta de fondo: si las reglas de la OIA han limitado el control que los ISP pueden ejercer sobre sus transmisiones a los edge providers, de forma tal que dichas normas constituyan un régimen per se de common carriage.

29. Verizon v. FCC, citado en nota 4, p. 52.

30. Resumidamente: I) que el hecho de que los edge providers no le soliciten servicios a los ISP (como lo exige la sección 20I (a) de la Ley de Comunicaciones) se refiere a una obligación del carrier y no a un requisito para determinar si dicho régimen es aplicable o no; 2) que la norma requiere que el carrier efectivamente le preste servicios al cliente, y por lo tanto no aplica si no hay cobro del ISP al edge provider, argumento inválido pues precisamente la imposición de la OIA obligaría a los ISP a no cobrarle a los edge providers por conexión con ellos, cuando eso es exactamente lo que ocurre en la actualidad; 3 ) que imposiciones normativas de no discriminación a entes que no son common carriers no es argumento suficiente para concluir que la FCC está autorizada para imponer obligaciones de common carriers a empresas reguladas que fueron expresamente excluidas de dicho régimen.

3I. Citado en nota 25. En dicho caso, la Corte Suprema sentenció que la FCC no tenía atribuciones, ni aun usando su jurisdicción supletoria, para obligar a empresas de televisión por cable a que no pudieran discriminar respecto del uso de ciertos canales de forma abierta, de lo transmitido en ellos, y de cobrarle a los usuarios por el uso de dichos canales, pudiendo efectuar un cobro «razonable» por el uso del resto de los canales.

32. Resumidamente: I) La FCC alega que en este caso, a diferencia de Midwest Video II, el contenido es entregado al usuario final sólo cuando éste lo requiera. La Corte rechaza este argumento, indicando que el acceso al contenido en este caso es idéntico al de Midwest Video II, y se refiere a la capacidad de los programadores/edge providers para que sus contenidos sean transmitidos a los usuarios finales si ellos lo desean. 2) La cantidad de contenidos transmitidos (limitados para televisión por cable, ilimitados para ISP) es irrelevante. 3) Respecto de la discreción editorial, no hay justificación para limitarla, sin importar el régimen de facultades empleado por la FCC; y, al igual que en Midwest Video II, la transferencia de control sobre los contenidos transmitidos afecta a los ISP como si fueran common carriers per se. 
La revisión que hace el Circuito D.C. la lleva a concluir que la OIA sí contiene normas que constituyen para los ISP un régimen per se de common carriage, específicamente:

- Respecto de la obligación de no discriminación impuesta a los prestadores de acceso a Internet fija, la Corte concluye inmediatamente que esta obligación reduce a dichos ISP al estatus de common carriers per se, señalando que la FCC fue incapaz de justificar su imposición y que esta obligación de no discriminación es sumamente amplia, siendo aplicable en cualquier circunstancia y respecto de todos los edge providers. ${ }^{33}$ El Circuito D.C. también rechaza el argumento de la FCC de que al permitir como excepción el que los ISP puedan implementar medidas razonables de administración de sus redes, dicha excepción impide configurar el régimen de common carriage per se, señalando que Verizon argumentó correctamente que esta excepción es nada menos que la misma que beneficia a los common carriers respecto de su derecho a rechazar clientes, ya sea porque no son los normalmente aceptados o porque su capacidad está saturada. En conclusión, la Corte indica que no ve un estándar de discriminación razonable en la OIA, esto es, la facultad de discriminar permitida a los common carriers mientras dicha discriminación no sea injusta o irracional, desde que la propia FCC argumentó que casos como el del «pago por prioridad» no cumplirían con el criterio de razonabilidad.

- Respecto de las normas de antibloqueo impuestas tanto a los prestadores de acceso a Internet fija como a Internet móvil, la FCC argumentó que dichas normas sólo establecen un piso de calidad de servicio (que los contenidos, aplicaciones y servicios de los edge providers sean «efectivamente usables») y el impedimento para los ISP de poder cobrar a los edge providers una tarifa por este piso mínimo de servicio, lo que no le impediría a los ISP cobrar una

33. La FCC intenta argumentar a partir del caso United States v. Southwestern Cable Co., 392 U.S. I 57 (1968), en el cual la Corte Suprema mantuvo una orden de la FCC que obligaba a las operadoras de televisión por cable a transmitir las señales de emisores locales cuando aquéllas importaban señales de otras emisoras que competían con las emisoras locales del área de servicio, señalando que entonces ese caso y éste serían idénticos. El Circuito D.C. rechaza este argumento. 
tarifa especial a cada edge provider por un nivel de servicio superior al piso mínimo. Pero la Corte no encontró que este argumento estuviera sustentado por el texto de la OIA, en la que no pueden diferenciarse los fundamentos de las normas antidiscriminación y antibloqueo, por lo que concluyó que en la práctica también sujetan a los ISP a un régimen per se de common carriage, lo que no es legal.

- Finalmente y respecto de las normas de transparencia, el Circuito D.C. concluyó que dichas normas no sujetan a los ISP a un régimen per se de common carriage. Y si bien Verizon no argumentó en este sentido, sí señaló que las normas de transparencia eran indivisibles de las normas de antibloqueo y antidiscriminación, por lo que la derogación de cualquiera de ellas debía resultar también en la derogación de las normas de transparencia. La Corte rechazó este argumento, acogiendo la defensa de la FCC que indicó que estas normas operaban de forma independiente al resto, por lo que la invalidez de las otras normas no afectaba la legalidad de las normas de transparencia.

Sentencia y voto de minoría. De forma muy somera, el Circuito D.C. resolvió dejar sin efecto todas las normas de antidiscriminación y de antibloqueo, pero mantuvo las normas de transparencia, y remitió la OIA nuevamente a la FCC para su revisión.

En todo caso, el fallo no fue unánime. El ministro Laurence Silberman concurrió parcialmente al fallo, al concluir con el resto de la Corte que la OIA en la práctica sujetaba ilegalmente a los ISP a un régimen de common carrier, pero fue mucho más allá: en su opinión, no hay disposición alguna en la Ley 1996, y menos en la sección 706, que le permita a la FCC dictar estas normas. El ministro Silberman también criticó a la Corte por hacer una mala lectura de la sección 706, al no exigirle a la FCC acreditar que cumplió con el fundamento legal para dictar las normas, esto es, el promover la competencia en los mercados locales de telecomunicaciones o dictar normas para eliminar barreras a la inversión en infraestructura. Ello porque, a su entender, respecto de la primera causal las normas debían estar destinadas a promover la competencia entre ISP (es decir, es requisito el que los ISP compitan en un mercado local de telecomunicaciones), mientras que respecto de la segunda norma, no se requiere competencia sino sólo barreras a las inversiones en 
infraestructura. Éste debió ser el fundamento para permitirle a la FCC dictar la OIA, pero el ministro Silberman criticó que la FCC en la OIA nunca identifica la existencia de este tipo de barreras anticompetitivas.

El ministro Silberman es mucho más incisivo: critica que la FCC se desvió de sus funciones regulatorias porque su argumento respecto del círculo virtuoso en Internet no tiene como objetivo el aumentar la competencia en el mercado de la banda ancha, sino que proteger a los edge providers de la eventual competencia en contenidos por los ISP. Al no reconocer esta situación, la Corte le estaría dando a la FCC poderes ilimitados para regular Internet.

Desde un punto de vista económico, el ministro Silberman le dio la razón al argumento de Verizon de que la FCC no acreditó la existencia de prácticas anticompetitivas o discriminatorias de los ISP para con los edge providers, señalando que la FCC siempre habla de prácticas que podrían suceder, no que efectivamente han sucedido. ${ }^{34}$ También argumenta que la FCC inventó el concepto del rol de "gatekeeper» ("portero») de los ISP y que el concepto de «monopolio de terminación» de los ISP para con los usuarios finales (la conexión de la última milla) no existe, y que no hay explicación alguna por la FCC de su significación económica. En la práctica, la posición del ministro Silberman es que estas posibilidades de discriminación por los ISP sólo pueden amparar normas antidiscriminatorias si es que los ISP tienen poder de mercado en el mercado de la banda ancha, y la dificultad para los usuarios de cambiarse de ISP no es evidencia de que éstos tengan poder de mercado (es sólo un factor).

\section{CONCLUSIONES}

Como se ha visto, la sentencia del Circuito D.C. está enfocada más en resolver el problema de si la FCC tiene o no atribuciones para dictar las normas antibloqueo y antidiscriminación en la OIA que regularían a los

34. La FCC plantea varios casos hipotéticos: los ISP podrían: a) Tener incentivos económicos para bloquear o perjudicar a edge providers específicos. b) Usar su poder para beneficiar a sus contenidos afiliados en perjuicio de los no afiliados. c) Actuar para beneficiar a edge providers que les han pagado, en perjuicio de aquellos que no les han pagado. d) Tener incentivos para incrementar sus utilidades cobrándoles a los edge providers. e) Suspender o negarse a incrementar su capacidad para «estrujar» el tráfico no priorizado. 
ISP, que en pronunciarse respecto a la legalidad o conveniencia intrínseca de reconocer normativamente el principio de neutralidad.

No obstante, existen bastantes antecedentes tanto en la OIA como en la sentencia que son indicativos de la doctrina prevalente respecto de los supuestos que justifican una imposición normativa del principio. En primer lugar, la FCC, por lo menos respecto de la OIA, invocó una teoría económica que es controversial y respecto de la cual no hay una conclusión definitiva: la del círculo virtuoso de las consecuencias de establecer obligaciones de antibloqueo y antidiscriminación que graven a los ISP; ello: I) facilita el desarrollo de contenidos, aplicaciones y servicios novedosos en Internet, lo que; 2) resulta en un aumento de la demanda de los usuarios por mejor y más rápido acceso a Internet, lo que; 3) resulta en mayor inversión de recursos por los ISP para ampliar y/o mejorar su infraestructura de redes. Verizon argumentó que esta conclusión de la FCC era errada y que, al contrario, la consecuencia de la imposición de reglas de antibloqueo y antidiscriminación será una menor innovación e inversión en redes, dado que las reglas resultarán en un aumento de costos, en impedir potenciales flujos de ingresos y en restringir la capacidad de los proveedores para proveer los servicios que los usuarios eventualmente requieran. ${ }^{35}$ Pero a Verizon le jugó en contra el hecho de que la FCC incluyera en la OIA, y luego en su defensa de la misma ante la Corte, varios ejemplos de prácticas anticompetitivas efectuadas por los ISP durante el último tiempo, las que si bien son escasas no fueron contrarrestadas por ejemplos de actuación de ISP que, teniendo el poder para efectuar discriminación, no lo han hecho. Además, y esto es un punto que la Corte resalta, el argumento de imposición normativa en base a discriminación económica hace necesario que quien discrimine tenga poder de mercado para hacerlo (argumento que también rescata el voto de minoría), y Verizon no argumentó que la falta de poder de mercado de los ISP podía tener como consecuencia que las imposiciones normativas en la OIA fueran caprichosas o arbitrarias, aunque obviamente ello no fue impedimento para que el Circuito D.C. anulara sustancialmente la OIA.

35. Verizon v. FCC, citado en nota 4, p. 43. Para un análisis detallado de los efectos de prácticas de bloqueo o discriminación en el mercado de acceso a Internet (y mercados relacionados), véase Van Schewick (2010). 
En segundo lugar, la aplicación del concepto de common carriage a Internet. No en vano, el principio de neutralidad también ha sido descrito como la aplicación del common carriage a Internet. ${ }^{36}$ Esta idea de reconocer que a estas alturas el acceso a Internet opera como una utility, ${ }^{37}$ es decir, como un suministro de un servicio público básico, como lo son la provisión de electricidad, agua potable o gas, ha ido tomando fuerza progresivamente con el pasar de los años, y se ha visto reflejada incluso en las diversas iniciativas que buscan reconocer el acceso a Internet como un derecho humano. No obstante, la institución del common carriage, como se ha visto, es una que sufre de defectos, entre ellos, su falta de definición precisa, su dificultad de implementar y fiscalizar, los fundamentos económicos de su configuración, etcétera. ${ }^{38}$ Está por verse si finalmente se implementarán las normas de neutralidad a través de un reconocimiento de que los proveedores de acceso a Internet son common carriers, pero por ahora todo parece indicar que, al menos en los Estados Unidos, ello no sucederá pues no parece haber incentivos para que la FCC reclasifique los servicios de acceso a Internet como servicios de transmisión, lo que le permitiría luego regularlos como common carriers.

Finalmente, siendo o no esta sentencia un hito en la historia del reconocimiento normativo del principio de neutralidad, todo parece indicar que la lucha de los ciudadanos y activistas por obtener dicho reconocimiento se ha puesto cuesta arriba. Si bien varios ISP han declarado y ratificado su intención de no efectuar discriminación de contenidos, el discurso sobre la neutralidad últimamente ha ido mutando hacia la posibilidad de permitir que los proveedores de acceso puedan negociar acuerdos privados con edge providers para establecer sistemas de acceso prioritario a los clientes de los ISP. Ello no sólo se ha visto en prácticas comerciales como las de Netflix con Comcast y Verizon, sino también en el anuncio de la FCC de volver a proponer normas que resguarden el principio de neutralidad, e incluso en los proyectos de ley que actualmente se discuten en la Unión Europea. ${ }^{39}$

\footnotetext{
36. Véase, por ejemplo, Wu (2010: 3 II) y Sandvig (2007).

37. Véase, por ejemplo, Crawford (20I3), Cooper (20I3) y Patel ((20I4).

38. Para un análisis detallado, véase Yoo (2013b).

39. En el Paquete Legislativo de Telecomunicaciones para el mercado único euro-
} 
Por lo pronto, y a partir de la sentencia, la FCC tiene las siguientes opciones:

- Puede volver a dictar las normas antibloqueo y antidiscriminación a partir de la sección 706 del título VII de la ley, pero ajustándose al espacio que le dejó el Circuito D.C. para que ellas no resulten en una aplicación de las normas aplicables a los common carriers, es decir, con una justificación legal distinta.

- En uso de sus facultades, puede recalificar a los ISP como servicios de transmisión y no de información, con lo cual quedarían sujetos válidamente a las obligaciones de common carrier establecidas en la Ley I996. Esta posibilidad se ve remota dado el costo político que tendría. La FCC toma sus decisiones a través de un directorio que es objeto de un fuerte lobby por parte de las empresas objeto de regulación, lo que ayudaría a entender por qué, luego de la invalidación que el Circuito D.C. hizo de la orden del 2005 que obligaba a Comcast a no interferir con el tráfico de aplicaciones peer-to-peer, la FCC no procedió inmediatamente a efectuar la reclasificación de dicha empresa, o de los ISP en general, a servicios de transmisión.

- Puede dejar sin efecto la OIA, o mantenerla sólo respecto de las normas de transparencia, y dictar nuevas normas pero que no sean de aplicación general, sino órdenes específicas para cada empresa regulada. Al parecer, esta sería la opción que tomaría la FCC. ${ }^{40}$

peo de las comunicaciones electrónicas actualmente en tramitación, las definiciones en la primera versión dejaban abierta la posibilidad de que los ISP pudieran discriminar, pero en la votación del pasado 3 de abril se introdujeron modificaciones al proyecto por las cuales se reconoce normativamente el principio de neutralidad, las que fueron aprobadas. Véase proyecto y modificaciones propuestas (especialmente véase Enmiendas 236 y 243 propuestas al artículo 23) en <http://www.europarl.europa.eu/sides/getDoc. do?pubref=-//ep//text+ta+p7-ta-20I4-O28 I +o+doc+xml+vo//es $>$.

40. Véase Gautham (20I4). Es importante destacar que lo que se sabe hasta ahora es que estas nuevas órdenes sí le permitirían a los ISP cobrarle a los edge providers por acceso preferente en la última milla a sus suscriptores, lo que ha significado duras críticas que anticipan que esto es el fin de la neutralidad de Internet propiamente tal. Véase, por ejemplo, Van Schewick (20I4). No obstante, las nuevas normas sólo serán publicadas a mediados de mayo de 2014 y, por lo tanto, a la fecha de cierre de este artículo, aún 


\section{REFERENCIAS}

Cannon, Robert (2003). "The legacy of the Federal Communications Commission's computer inquiries». Federal Communications Law Journal, 55 (2): 167-205.

CraWford, Susan (2013). Captive audience: The telecom industry and monopoly in the New Gilded Age. New Haven: Yale University Press.

CoOper, Steve (2013). "The Internet is a 2 Ist Century utility and we deserve better». Forbes, 29 de enero, disponible en <http://www.forbes.com/sites/stevecooper/20 I3/OI/29/the-internet-is-a-2 Ist-centuryutility-and-we-deserve-better/>.

FCC (Federal Communications Commission) (2002). In re inquiry concerning high-speed access to the Internet over cable and other facilities, I7 F.C.C.R. 4798 (2002), disponible en <https://apps.fcc.gov/ edocs_public/attachmatch/FCC-02-77AI.pdf $>$.

-. (20I0). Open Internet Order, disponible en <http://hraunfoss.fcc. gov/edocs_public/attachmatch/FCC-IO-20IAI_Rcd.pdf>.

Gautham, Nagesh (20I4), «FCC to propose new 'net neutrality' rules». The Wall Street Journal, 23 de abril, disponible en <http://online.wsj. com/news/articles/SBIo00I4240527023045 I 87045795 I 99634 I 635 0296 ? $\mathrm{mod}=\mathrm{LS}$ I $>$ (enlace pagado).

Gross, Grant (20I4). «FCC's new net neutrality proposal: What do we really know?». PC World, 28 de abril, disponible en <http://www.

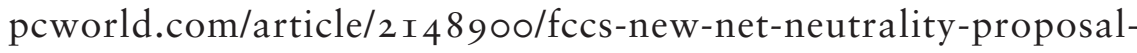
what-do-we-really-know.html>.

Owen, Bruce M. y Gregory L. Rosston (2006). «Local broadband access: primum non nocere or primum processi? A property rights approach». En Thomas Lenard y Randolph L. May (eds.), Net neutrality or net neutering. Should broadband Internet services be regulated? Nueva York: Springer.

Patel, Nilay (20I4). «The Internet is fucked». The Verge, 25 de febre-

se desconoce su contenido. Véase Gross (20I4). Al cierre de este artículo, la FCC había subido a su página web su Propuesta de Norma para Proteger y Promover una Internet Abierta ( Notice of Proposed Rulemaking in the Matter of Protecting and Promoting the Open Internet»), disponible en <http://transition.fcc.gov/Daily_Releases/Daily_Business/20I4/dbo5 I 5/FCC-I4-6IAI.pdf>. 
ro, disponible en $<$ http://www.theverge.com/20I $4 / 2 / 25 / 543$ I $382 /$ theinternet-is-fucked $>$.

SANDVIG, Christian (2007). "Network neutrality is the new common carriage». The Journal of Policy, Regulation and Strategy for Communications, Information and Media, 9 (2/3): I3 6-I 47.

VAN SCHEWICK, Barbara (20I0). Internet architecture and innovation. Cambridge: The MIT Press.

-. (20I4) «The FCC changed course on network neutrality. Here is why you should care», disponible en <http://netarchitecture.org/20I4/o4/ the-fcc-changed-course-on-network-neutrality-here-is-why-youshould-care/>.

Wu, Tim (2003). "Network neutrality, broadband discrimination». Journal of Telecommunications and High Technology Law, 2 (I): I 4 I-I 76 .

-. (2010). The master switch: The rise and fall of information empires. Nueva York: Alfred A. Knopf.

Yoo, Christopher (20I3a). «Libertad de expresión y el mito de Internet como una experiencia no intermediada». Revista Chilena de Derecho y Tecnología, 2 (I): I I-I I I.

-. $\left(20 \mathrm{I}_{3} \mathrm{~b}\right)$. «Is there a role for common carriage in an Internet-based world?» Houston Law Review, 5 I (2): 545-608.

\section{SOBRE EL AUTOR}

Martín Mois Freiwirth es abogado. Licenciado en Ciencias Jurídicas y Sociales por la Universidad de Chile y Master of Law por la Universidad de Pennsylvania. Actualmente se desempeña como asociado senior en el estudio Aninat Schwencke \& Cía. Su correo electrónico es $<$ mmois@asyc.com>.

Este trabajo fue recibido el 29 de abril de 20 I 4 y aprobado el I 2 de junio de 2014 . 
